\title{
A Case of Coronary Artery Dissection After Aortic Replacement in Acute Type A Aortic Dissection
}

\author{
Sun Hee Park, MD ${ }^{1}$, Hun Sik Park, MD¹, Jang Hoon Lee, MD ${ }^{1}$, Hyeon Min Ryu, MD \\ Jae Hee Kim, MD ${ }^{1}$, Won Suk Choi, MD ${ }^{1}$, Kyun Hee Kim, MD ${ }^{1}$ and Gun Jik Kim, MD² \\ ${ }^{1}$ Departments of Internal Medicine and ${ }^{2}$ Thoracic and Cardiovascular Surgery, Kyungpook National University Hospital, \\ Daegu, Korea
}

\section{ABSTRACT}

A 59-year-old woman was transferred to our institution with a diagnosis of acute type A aortic dissection. During aortic replacement surgery, the dissection had not extended to the orifice of the left coronary artery. However, ST segment elevation was observed on an electrocardiogram monitor immediately postoperatively. An emergent coronary angiogram showed almost complete collapse of the lumen of the left coronary artery due to pulsatile compression of the false lumen, which was caused by extension of the aortic dissection. Percutaneous coronary intervention (PCI) was performed with placement of stents in the left anterior descending artery (LAD) and left circumflex artery. Coronary angiography and intravascular ultrasound performed 45-days after PCI showed significant instent restenosis (ISR) at the proximal portion of the LAD and residual coronary artery dissection of the diagonal branch. Repeat balloon angioplasty was performed at the site of the ISR. A follow-up coronary angiogram 8-months after the PCI showed no evidence of ISR. (Korean Circ J 2009;39:428-433)

KEY WORDS: Aortic diseases; Myocardial infarction; Cordiovascalar surgical procedures; Percutaneous transluminal coronary angioplasty.

\section{Introduction}

Malperfusion of coronary arteries due to acute Stanford type A aortic dissection is particularly problematic because it may be obscured by myocardial ischemia or infarction. ${ }^{1)}$ Acute myocardial infarction due to extension of an acute Stanford type A aortic dissection is an infrequent, but devastating event. ${ }^{23)}$ Several case reports of Stanford type A aortic dissections in combination with myocardial infarctions present prior to surgery have been published. ${ }^{2-6)}$ We report here an interesting case involving an acute aortic dissection in which coronary malperfu-

Received: February 16, 2009

Revision Received: May 28, 2009

Accepted: June 10, 2009

Correspondence: Hun Sik Park, MD, Department of Internal Medicine, Kyungpook National University Hospital, 200 Dongdeok-ro, Jung-gu, Daegu $700-$ 721, Korea

Tel: 82-53-420-5527, Fax: 82-53-426-2959

E-mail: hspark@knu.ac.kr

(a) This is an Open Access article distributed under the terms of the Creative Commons Attribution Non-Commercial License (http://creativecommons. org/licenses/by-nc/3.0) which permits unrestricted non-commercial use, distribution, and reproduction in any medium, provided the original work is properly cited. sion progressed postoperatively.

\section{Case}

A 59-year-old woman was admitted to the emergency room of a local hospital with the sudden onset of severe chest pain radiating to her back. She had a history of hypertension and a cerebrovascular accident. At the time of presentation, she had a drowsy mental status. The cardiac enzymes were elevated on baseline laboratory testing. A chest film showed mediastinal widening (Fig. 1). The initial electrocardiogram (ECG) showed a normal sinus rhythm without significant ST-T changes (Fig. 2). An emergent chest CT scan was performed under the suspicion of an acute aortic dissection, which showed an acute Stanford type A aortic dissection (Fig. 3). The patient was then transferred to our institution for repair of the aortic dissection. She was hemodynamically unstable.

Emergent ascending aortic replacement surgery using a graft was performed with the patient under general anesthesia using a median sternotomy. Upon opening the pericardium, a hemopericardium was noted. The aortic root was incised, and an intimal tear was identified just above the sinus of Valsalva. The dissection had not 
extended to the orifice of the left coronary artery. We carried out an ascending aortic replacement with a 28-

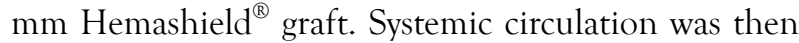
restarted. ST segment elevation was observed on the ECG monitor immediately postoperatively. Sedation and mechanical ventilation were maintained, and she was transferred from the operating room to the intensive care unit (ICU).

In the ICU her blood pressure and cardiac output remained low in spite of sufficient inotropic support. An ECG showed ST segment elevation in leads II, III, and $\mathrm{aVF}$, and in the precordial leads, suggesting broad myocardial ischemia (Fig. 4). In addition, transthoracic echocardiography showed regional wall motion abnormalities in the territories of the left anterior descending artery

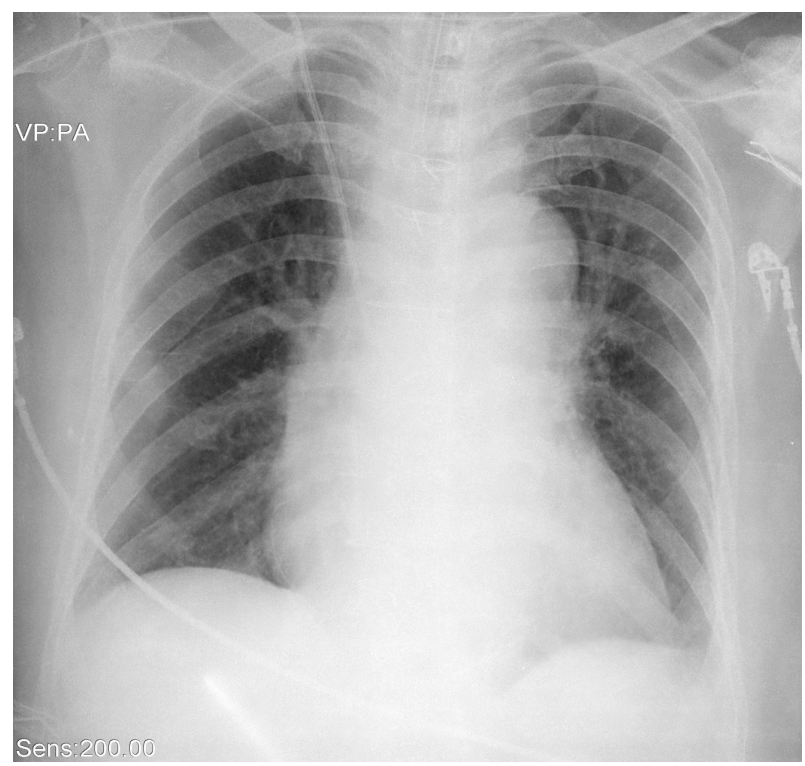

Fig. 1. Chest $\mathrm{X}$-ray showing mediastinal widening.
(LAD) and left circumflex artery (LCx), and moderately decreased LV systolic function. These results suggest coronary artery malperfusion, probably caused by progression of the aortic dissection into the left coronary artery. Accordingly, emergent coronary angiography was performed 90 minutes postoperatively.

Coronary angiography revealed no significant luminal narrowing in the right coronary artery, but almost complete collapse of the lumen of the LAD (Fig. 5A). After forceful contrast injection into the LAD, the collapsed lumen was reopened, but it subsequently recollapsed without forceful contrast injection. A similar finding was observed in the LCx (Fig. 5B). We reasoned that this lifethreatening coronary artery occlusion was caused by pulsatile compression of the false lumen of the left coronary artery dissection. A zotarolimus-eluting stent was deployed in the mid-portion of the LAD and a bare metal stent $\left(\right.$ Tsunami ${ }^{\circledR}, 3.5 \times 30 \mathrm{~mm}$ ) was deployed in the proximal portion of the LAD. Three bare metal stents (Driver ${ }^{\circledR}$, $3.0 \times 30 \mathrm{~mm}$; Tsunami ${ }^{\circledR}, 3.0 \times 30 \mathrm{~mm}$; and Driver $^{\circledR}, 3.0$ $\times 18 \mathrm{~mm}$ ) were successfully deployed from the distal portion to the proximal portion of the LCx. However, coronary blood flow was not improved. Thus, direct stenting at the left main stem, and a bare metal stent (Driver ${ }^{\circledR}$, $3.5 \times 18 \mathrm{~mm}$ ) was successfully deployed. The final angiogram of the left coronary artery showed Thrombolysis In Myocardial Infarction (TIMI) flow grade 3 in the LAD and LCx.

Follow-up coronary angiography was performed 45 days after percutaneous coronary intervention (PCI) because the patient remained unstable. Dyspnea was New York Heart Association (NYHA) class III/IV and followup two-dimensional echocardiogram (2D-UCG) revealed a severe decrease in LV systolic function. Follow-up coronary angiogram showed significant in-stent reste-

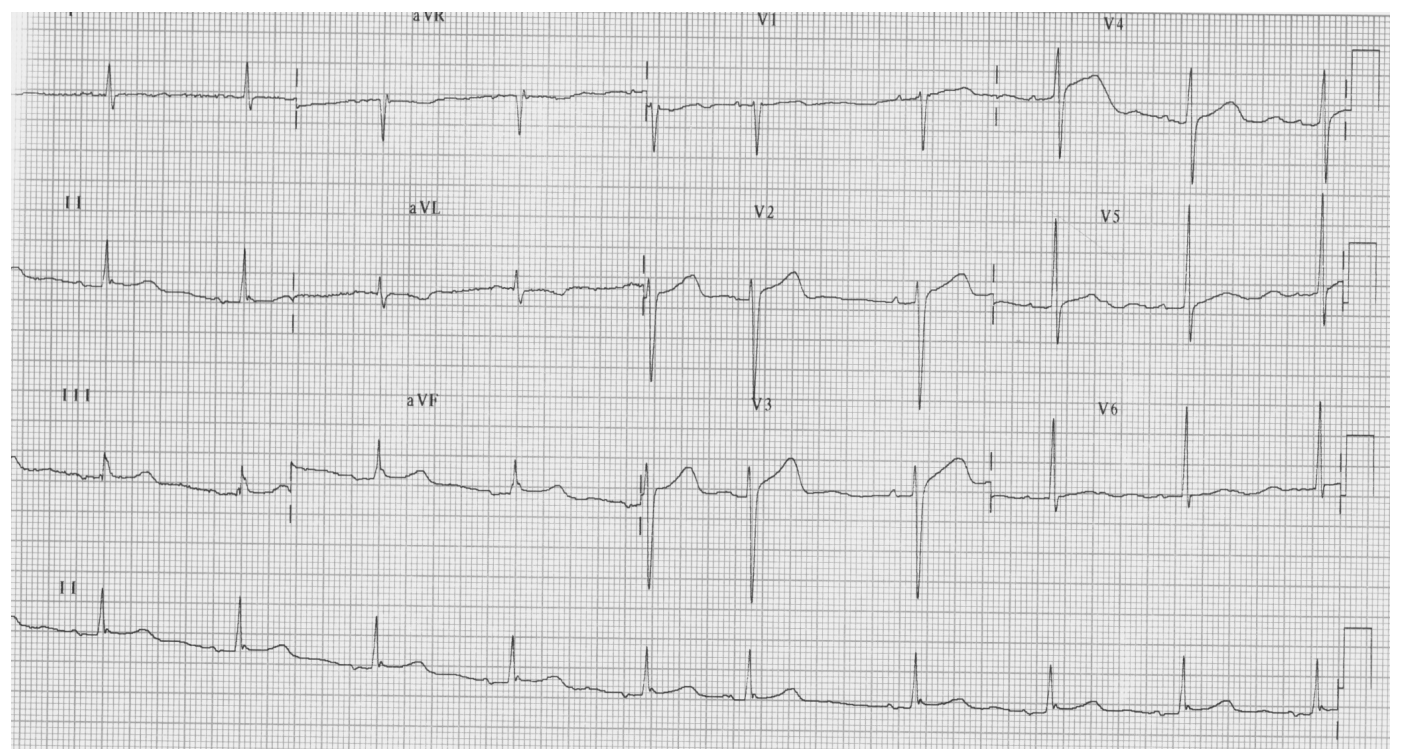

Fig. 2. Initial electrocardiogram showed normal sinus rhythm with a premature atrial complex. There were no significant ST-T changes. 

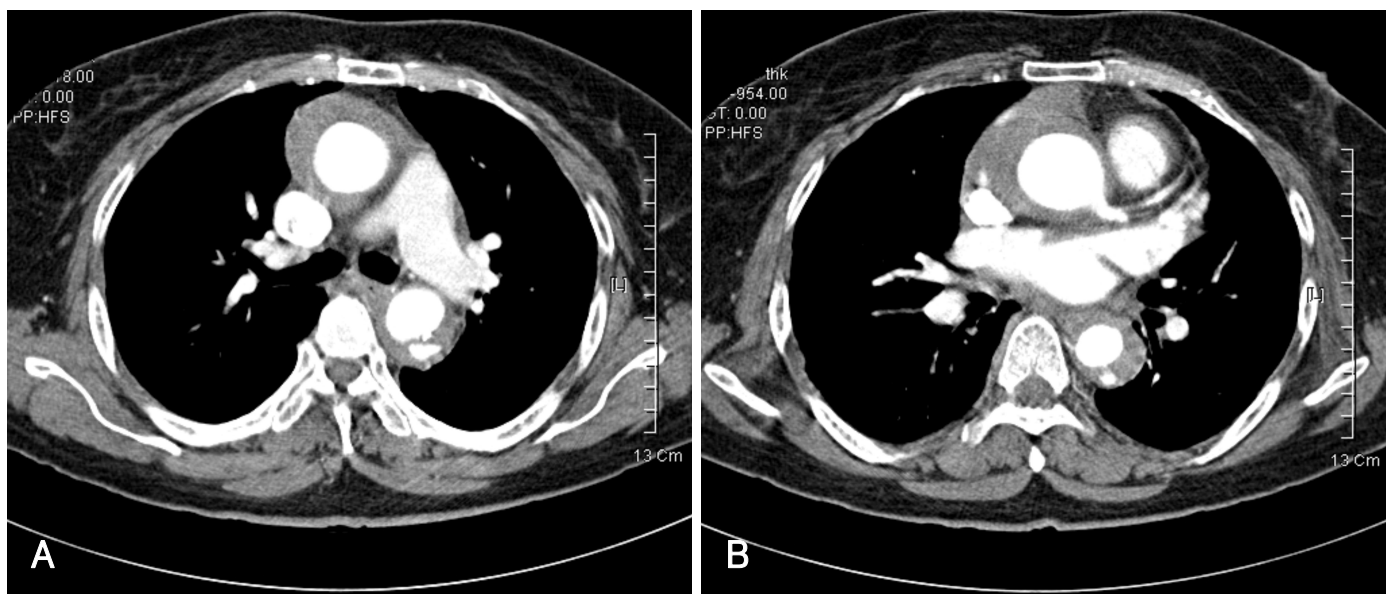

Fig. 3. Emergent computed tomogram of the chest showing an acute Stanford type A aortic dissection (A). The left coronary artery had good patency and no dissection (B).

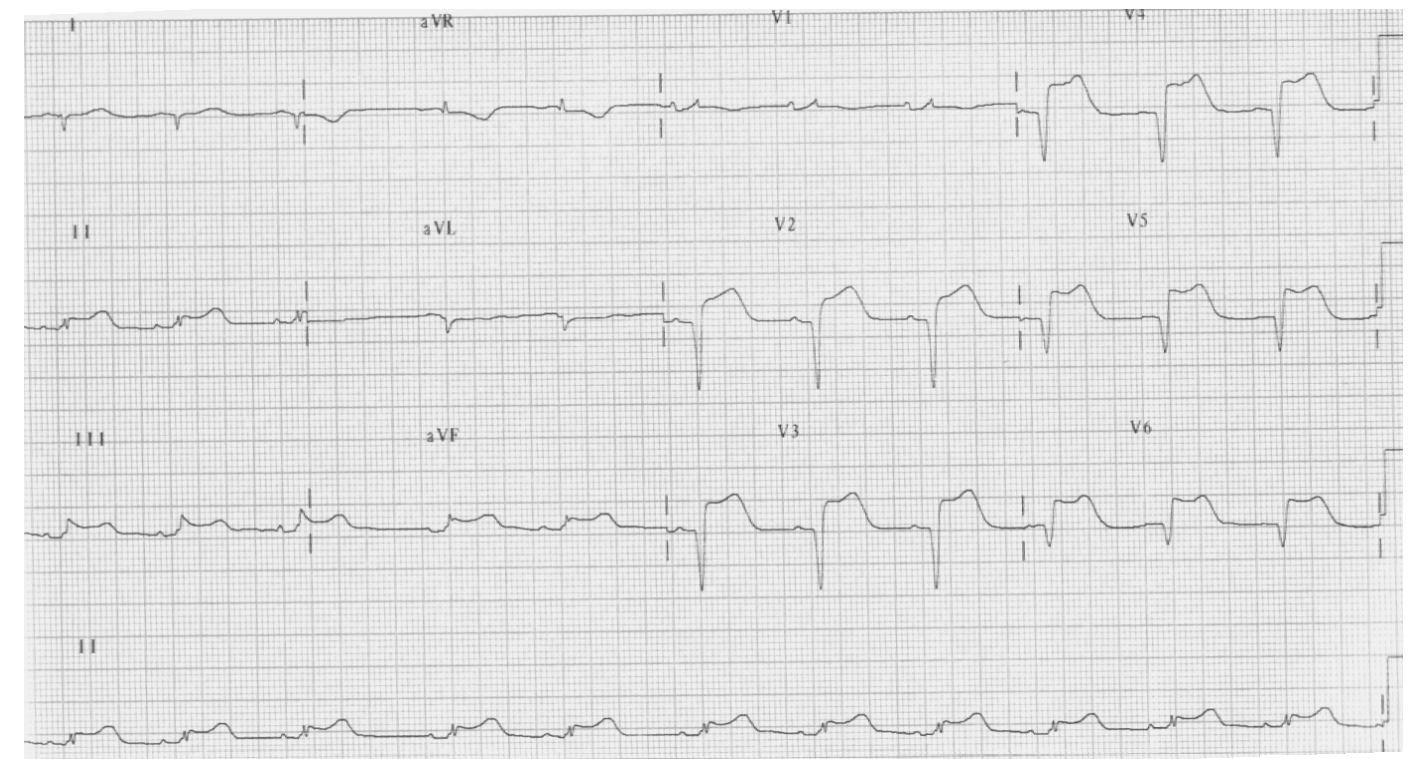

Fig. 4. Electrocardiography demonstrated ST segment elevation in leads II, III, and aVF, and in the precordial leads immediately postoperatively.

nosis at the proximal portion of LAD (Fig. 6). An intravascular ultrasound (IVUS) study showed the remaining coronary artery dissection in the diagonal branch of the LAD (Fig. 7). Repeat balloon angioplasty using a $4.0 \times$ $20 \mathrm{~mm}$ balloon (Apollo ${ }^{\circledR}$ ) was performed at the site of in-stent restenosis. The final angiogram of the LCA showed TIMI flow grade 3 in the LAD and LCx. Her clinical course following the repeat PCI was stable and uneventful. The patient was discharged from hospital 51 days after the surgical repair. A follow-up coronary angiogram was performed 8 months after the PCI and showed no significant in-stent restenosis (Fig. 8).

\section{Discussion}

Coronary malperfusion associated with aortic dissection is relatively rare, but when it occurs, it is generally fatal. ${ }^{2) 7}$ Retrograde dissection of the aortic root reaching the coronary ostium was noted in 7\% of necropsies according to a previous study. ${ }^{8)}$ In acute Stanford type A aortic dissection, acute myocardial ischemia and infarction due to this mechanism are severe complications, which potentially lead to irreversible myocardial damage. Therefore, coronary artery ischemia and concomitant coronary artery bypass grafting (CABG) have been reported to be significant risk factors of postoperative mortality. ${ }^{9}$ ) To salvage such moribund patients, aggressive coronary revascularization concomitant with aortic repair is essential. ${ }^{2)}$

In the present case, myocardial infarction occurred immediately postoperatively, but the patient showed no previous signs of myocardial ischemia prior to the operation. Generally, myocardial ischemia caused by acute aortic dissection is apparent soon after its onset, and signs of myocardial ischemia are present prior to surgery. ${ }^{2-6)}$ When a Stanford type A aortic dissection extends 


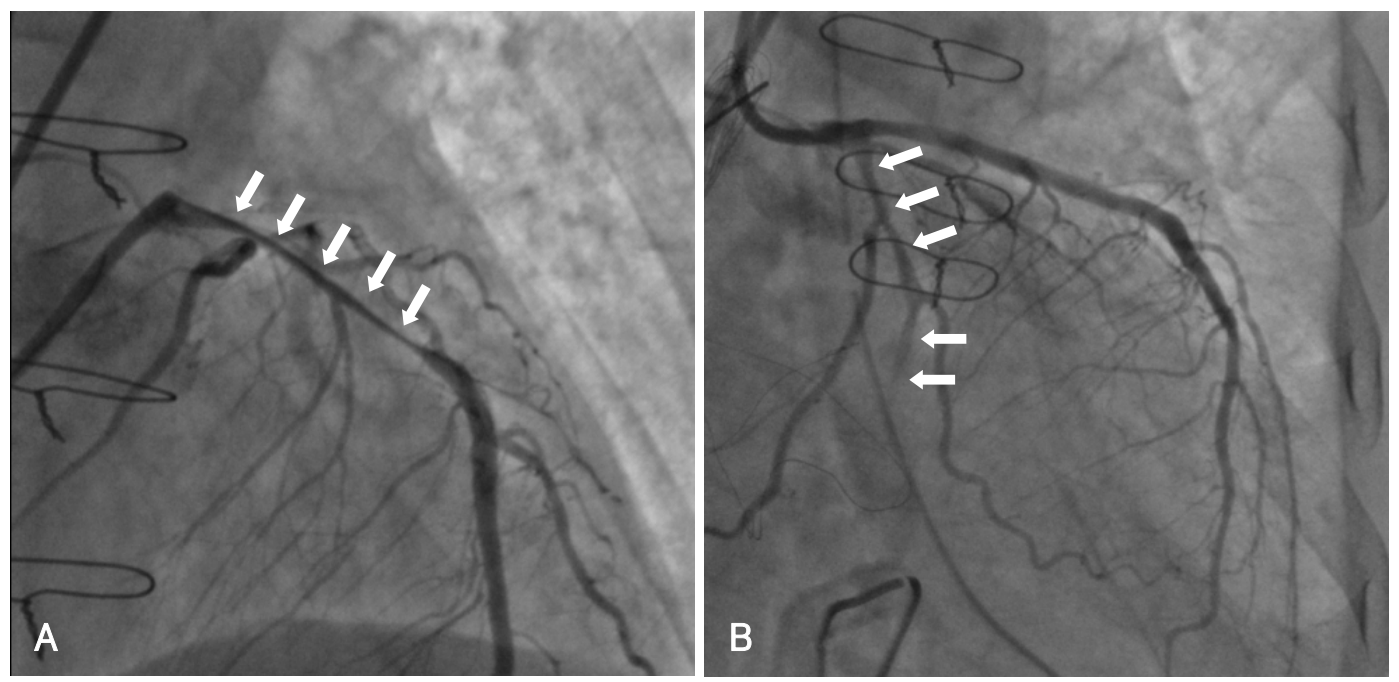

Fig. 5. Postoperative emergent coronary angiogram showing almost complete collapse of the lumen of the left anterior descending artery (A). After forceful contrast injection into the left anterior descending artery, the lumen of the collapsed left anterior descending artery which had been reopened, subsequently recollapsed (arrows). The dissection encircled the affected coronary ostium and the observed malperfusion was produced by direct coronary blood flow obstruction. Similar findings were obtained in the left circumflex artery (arrows) (B).
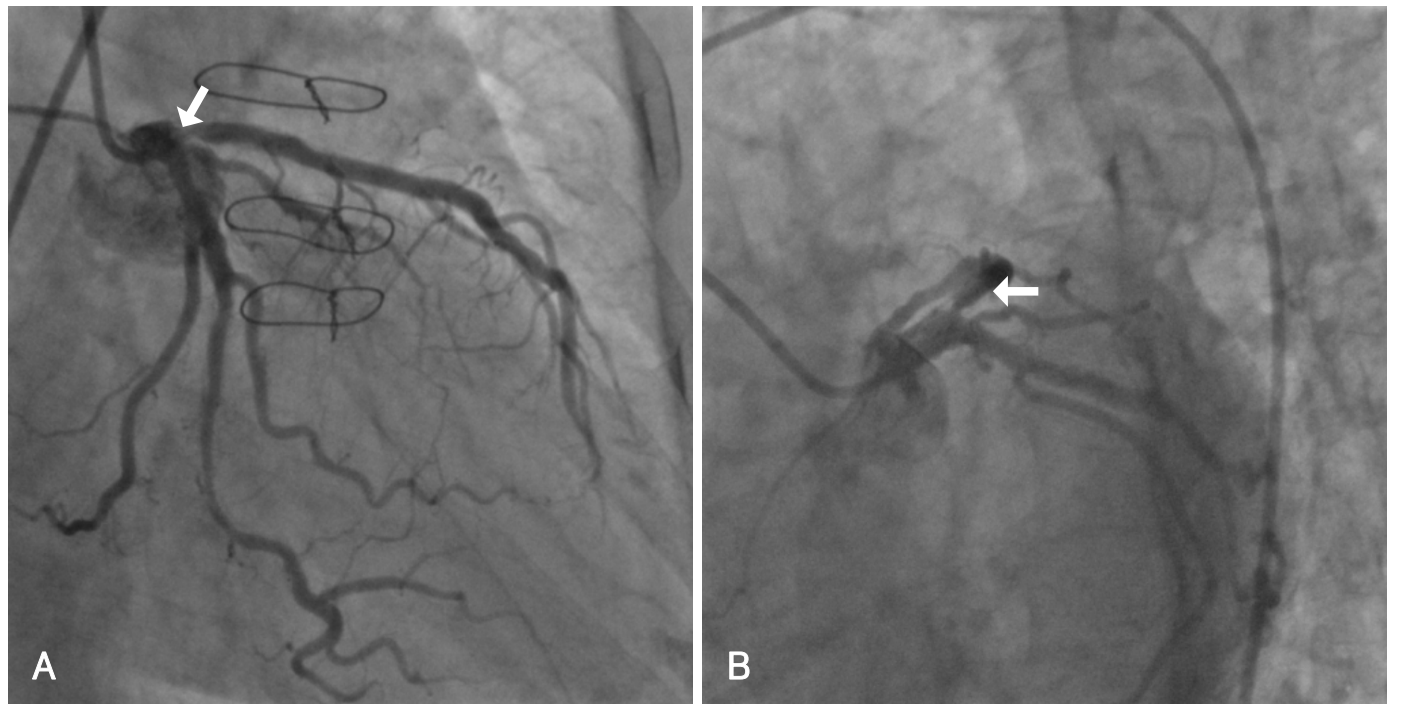

Fig. 6. Follow-up coronary angiogram performed 45-days after percutaneous coronary intervention showing significant in-stent restenosis at the proximal portion of the left anterior descending artery in the anteroposterior caudal (arrow) (A) and in the left anterior oblique caudal views (arrow) (B).

to the left coronary artery after aortic replacement surgery, catastrophic hemodynamic changes occur, which frequently result in sudden death. ${ }^{10)}$ Treatment of a coronary artery dissection depends on its severity. Three main types of coronary lesion can be caused by ascending aortic dissection, depending on the extent: type A, ostial dissection; type $\mathrm{B}$, dissection with a coronary false channel; and type $\mathrm{C}$, circumferential detachment with an inner intussuscepted cylinder. ${ }^{3)}$ However, these types are not easily differentiated. In the present case, the coronary artery ostium appeared intact, as in a type A coronary dissection. Indeed the following sequence of events may have occurred. A fragile intima may have been damaged by a needle, arterial blood flowed into the false lumen, and coronary artery dehiscence resulted if management of the false lumen was inadequate. Accordingly, type A coronary artery dissection progresses to type $\mathrm{B}$ or $\mathrm{C}$ dissection, and blood from the aortic false channel progresses into the coronary ostium and creates an extension of the false lumen into the coronary artery. The mechanism of coronary flow impairment was attributed to compression in diastole of the true lumen by obstructing the false channel. Finally, the dissection may have encircled the affected coronary ostium and caused malperfusion by directly obstructing coronary blood flow.

In types $\mathrm{B}$ and $\mathrm{C}$ dissections, it is not difficult to decide whether to undertake concomitant CABG. However, the role of concomitant CABG in type A dissection remains controversial. ${ }^{3)}$ In the present case, myocardial ischemia should have been suspected when circulatory 


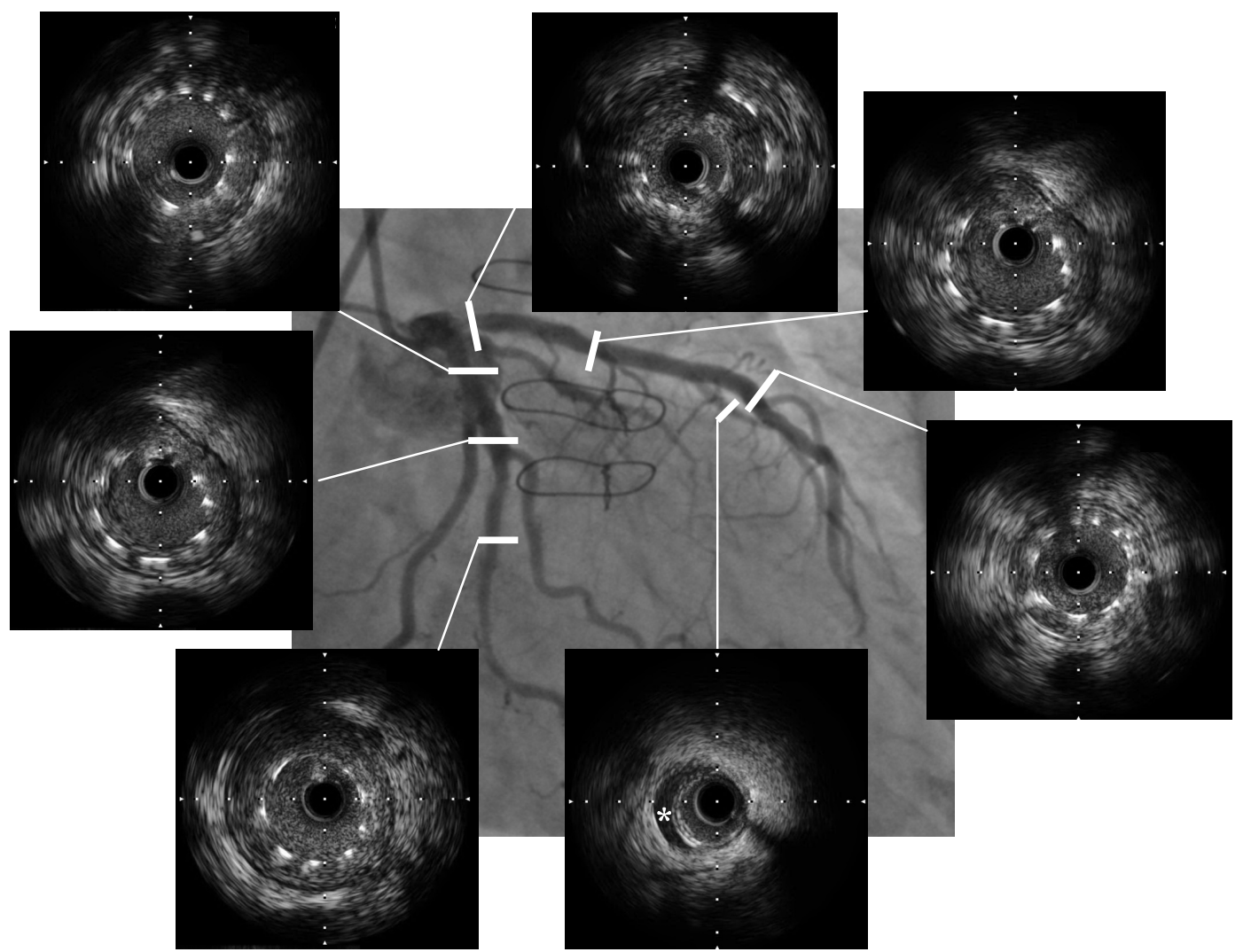

Fig. 7. The intravascular ultrasound study showed significant in-stent restenosis in the proximal portion of the left anterior descending artery and remaining coronary artery dissection in the diagonal branch of the left anterior descending artery $\left({ }^{*}\right)$. There was no significant instent restenosis or remaining dissection of the left circumflex artery.

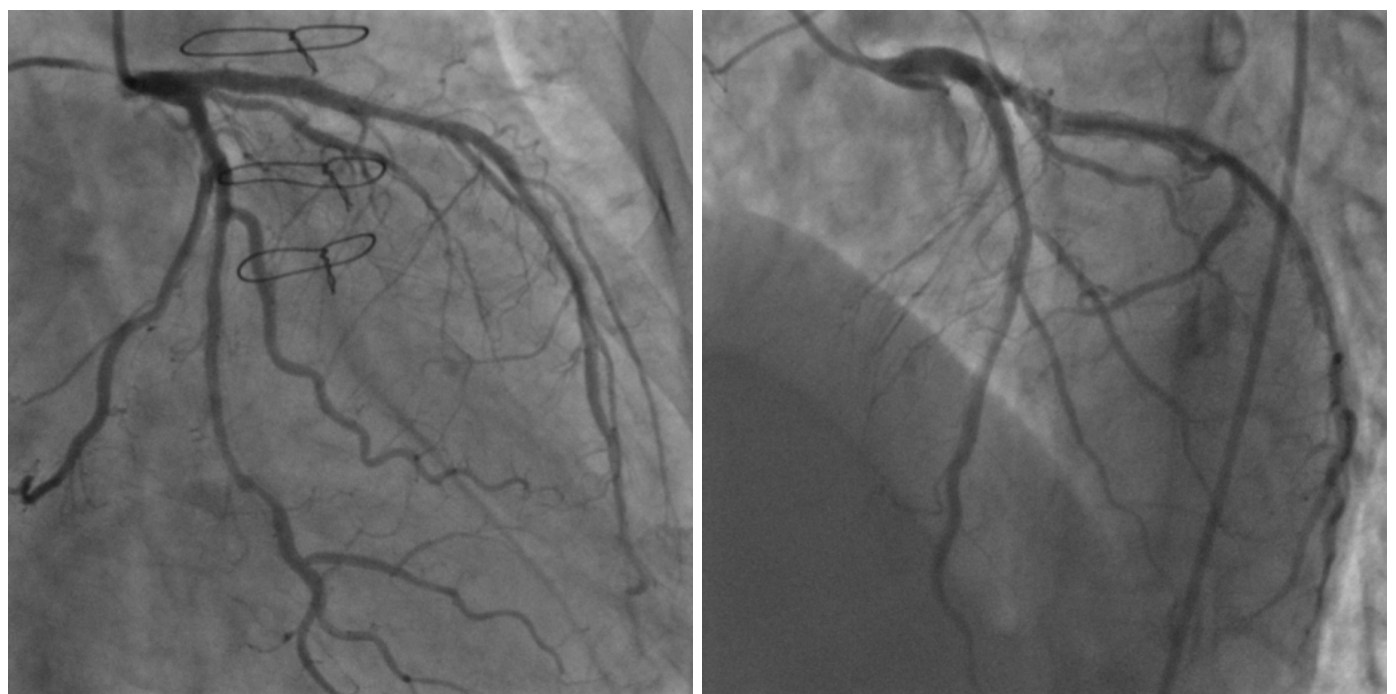

Fig. 8. Follow-up coronary angiogram performed 8 months after percutaneous coronary intervention showing no significant in-stent restenosis.

collapse was observed after terminating cardiopulmonary bypass, and at this stage immediate revascularization by concomitant CABG or PCI was necessary. Although no definite method for treating an infarcted myocardium has been established, the restoration of coronary circulation as quickly as possible appears to be important. In the present case, we decided to perform immediate revascularization by emergent $\mathrm{PCI}$ rather than $\mathrm{CABG}$ as an optional approach because pulsatile compression of the left coronary artery by coronary arterial dissection resulted in a life-threatening situation at the time of coronary angiography. It has been reported that an IVUS study can promptly diagnose direct extension of a dissecting flap into the coronary arteries and subsequent coronary malperfusion in potentially fatal conditions. ${ }^{11)}$

Once coronary malperfusion has occurred, the success 
of myocardial salvage depends on how quickly the malperfusion is recognized. Transesophageal echocardiography is one of the most sensitive and specific modalities for diagnosing acute aortic dissection and coronary malperfusion, even during surgery. ${ }^{12)}$ On the other hand, the role of coronary angiography before emergency repair of an acute aortic dissection remains controversial. ${ }^{13)}$ Although coronary artery malperfusion associated with acute aortic dissection after surgery is relatively rare, ${ }^{14)}$ it is possible for acute Stanford type A aortic dissections. Furthermore, an immediate decision is required to perform additional CABG if required. In the present case, emergent PCI may have been an optional approach given the critical, unstable patient postoperatively. An IVUS study could also provide additional information about the cause of coronary artery malperfusion.

\section{REFERENCES}

1) Nah DY, Park KU, Kim SH. Acute proximal aortic dissection associated with ST segment elevation on electrocardiography. Korean Circ J 2004;34:795-8.

2) Kawahito K, Adachi H, Murata S, Yamaguchi A, Ino T. Coronary malperfusion due to type A aortic dissection: mechanism and surgical management. Ann Thorac Surg 2003;76:1471-6.

3) Neri E, Toscano T, Papalia U, et al. Proximal aortic dissection with coronary malperfusion: presentation, management, and outcome. J Thorac Cardiovasc Surg 2001;121:552-60.

4) Kwon YS, Lee HS, Shin JK, et al. A case of left main coronary stenting for acute myocardial infarction complicated by ascending aortic dissection. Korean Circ J 2004;34:1210-5.
5) Horszczaruk GJ, Roik MF, Kochman J, et al. Aortic dissection involving ostium of right coronary artery as the reason of myocardial infarction. Eur Heart J 2006;27:518.

6) Weber M, Kerber S, Rahmel A, Breithardt G, Diallo S, Bocker W. Acute thoracic aortic dissection with occlusion of the left coronary artery. Herz 1997;22:104-10.

7) Geirsson A, Szeto WY, Pochettino A, et al. Significance of malperfusion syndromes prior to contemporary surgical repair for acute type A dissection: outcomes and need for additional revascularizations. Eur J Cardiothorac Surg 2007;32:255-62.

8) Hirst AE Jr, Jones VJ Jr, Kime SW Jr. Dissecting aneurysm of the aorta: a review of 505 cases. Medicine 1958;37:217-79.

9) Kawada T, Okada Y, Aiba M, et al. Changing predictors of postoperative mortality in acute type A aortic dissection: is only coronary artery compromise significant? Jpn J Thorac Cardiovasc Surg 2001;49:347-54.

10) DeSanctis RW, Doroghagi RM, Austen WG, Buckley MJ. Aortic dissection. N Engl J Med 1987;317:1060-7.

11) Na SH, Youn TJ, Cho YS, et al. Acute myocardial infarction caused by extension of a proximal aortic dissection flap into the right coronary artery: an intracoronary ultrasound image. Circulation 2006;113;e669-71.

12) Ohuchi H, Kyo S, Matsumura M, et al. Global myocardial ischemia as a complication of an acute type A aortic dissection: rapid diagnosis of a case by transesophageal echocardiography. Jpn Circ J 2000;64:533-6.

13) Penn MS, Smedira N, Lytle B, Brener SJ. Does coronary angiography before emergency aortic surgery affect in-hospital mortality? J Am Coll Cardiol 2000;35:889-94.

14) Funatsu T, Fukuda H, Takeuchi M, Masai M, Kawano S, Abe K Progression of left coronary artery dissection during and after aortic replacement in acute type A aortic dissection: a case report. Ann Thorac Cardiovasc Surg 2007;13:209-12. 